\title{
PRODUTIVIDADE DO MILHO EM UM SISTEMA SILVIAGRÍCOLA NOS CAMPOS GERAIS, PR
}

\section{YIELD OF MAIZE GROWN IN THE ALLEY CROPPING SYSTEMS IN THE CAM- POS GERAIS, PR}

\author{
Vanderley PORFIRIO-DA-SILVA ${ }^{1}$ \\ Anibal de MORAES ${ }^{2}$ \\ José Luis MOLETTA ${ }^{3}$ \\ Adelino PELISSARI ${ }^{2}$ \\ Jeferson DIECKOW ${ }^{2}$ \\ Edilson Batista de OLIVEIRA ${ }^{1}$
}

\begin{abstract}
RESUMO
Em sistemas silviagrícolas a produtividade de grãos pode ser afetada por vários fatores, o resultado pode ser nulo, de redução, ou, de aumento da produtividade. O objetivo deste trabalho foi comparar a produtividade do milho plantado solteiro e em integração com árvores espaçadas em $14 \mathrm{~m} \times 3 \mathrm{~m}$ (silviagrícola), e detectar efeitos resultantes da interação entre arvores e lavoura no sistema silviagrícola estudado. A produtividade do milho (cultivar IPR-114) foi avaliada em três tratamentos com seis repetições e o incremento de madeira, durante o período de cultivo do milho, foi obtido com o auxílio de monitoramento dendrométrico. Por meio da análise de variância não foi detectado efeito dos tratamentos $(P>0,05)$ na produtividade do milho. A produtividade média de grãos foi de $4.247,9 \pm 256,1 \mathrm{~kg} \mathrm{ha}^{-1}$ para o milho solteiro e de 4.554,99 $\pm 409,9 \mathrm{~kg} \mathrm{ha}^{-1}$ para o silviagrícola. No silviagrícola, o milho ocupou $71 \%$ da área enquanto que as árvores ocuparam $29 \%$. A razão do efeito da interação $(\square)$ entre os componentes lavoura e árvore foi de $6,7 \%$, o que denota favorecimento do sistema silviagrícola para a produtividade do milho.

Palavras-chave: agroflorestal; integração lavoura-floresta; IPR-114; Eucalyptus dunnii; Schinus terebinthifolius; Grevillea robusta
\end{abstract}

\section{ABSTRACT}

In alley cropping systems grain yields can be affected by several factors, the result can be null, or reduction or increase productivity. The objective of this research was to compare the productivity of corn grown sole and integrated with trees spaced at $14 \mathrm{~m} \times 3 \mathrm{~m}$ (alley cropping systems), and detect effects of the interaction between trees and crops in the system. The productivity of maize (cultivar IPR-114) was evaluated in three treatments with six repetitions, and timber increase during the cultivation of maize was obtained with the aid of dendrometric monitoring. Through the variance analysis was not found treatment effects $(P>0.05)$ in corn yields. The average yield of corn was $4247.9 \pm 256.1 \mathrm{~kg} \mathrm{ha}^{-1}$ for corn sole and $4554.99 \pm 409.9 \mathrm{~kg}$ ha- 1 for alley cropping. In alley cropping systems, corn occupied $71 \%$ of the area while the trees occupied $29 \%$. The effect of the interaction (I) between the components both crop and tree was $6.7 \%$, which shows advantage to alley cropping system to the productivity of corn.

Key words: agroforestry; tree-crop integration; IPR-114; Eucalyptus dunnii; Schinus terebinthifolius; Grevillea robusta

\footnotetext{
${ }^{1}$ Embrapa Florestas, Estrada da Ribeira, km 111, C.P. 319, CEP 83411-000, Colombo, PR E-mail: porfirio@cnpf.embrapa.br, edilson@cnpf.embrapa.br

2Universidade Federal do Paraná, C. P. 19061, CEP 81.531-990, Curitiba, PR. E-mail: anibalm@ufpr.br, linopeli@hotmail.com, jefersondieckow@ufpr.br

${ }^{3}$ Instituto Agronômico do Paraná, Est. Exp. Faz. Modelo, C. P. 129, CEP 84.001-970, Ponta Grossa, PR
} 
PORFÍRIO-DA-SILVA, V. et al. Produtividade de milho em um sistema...

\section{INTRODUÇÃO}

As produtividades de lavouras integradas com árvores (silviagrícola) podem aumentar ou diminuir dependendo do arranjo espacial (Reynolds et al., 2007; Reis et al., 2007), da mitigação da competição por umidade (Miller \& Pallardy, 2001), da dimensão e manejo das árvores no tempo (Burner et al., 2009; Devkota et al, 2009; Reis et al., 2007) e da espécie agrícola (Kummel, 2009), entre outros fatores.

A redução na produtividade dos cultivos agrícolas plantados entre aléias tem sido atribuída à sombra das árvores, tanto nas condições tropicais, quanto nas regiões temperadas (Burner et al., 2009). Adicionalmente, a competição por umidade do solo tem sido relatada como o principal fator limitante de cultivos entre renques arbóreos, tanto de regiões semi-áridas tropicais (Ong \& Huxley, 1996 como de regiões temperadas (Jose et al. 2000).

$O$ efeito de interação entre plantas pode ser positivo (favorecimento), neutro ou negativo (competição), e que depende de diversos fatores ecológicos (Callaway \& Walker, 1997; Gillespie et al., 2000), as interações entre as árvores e a lavoura associadas em um sistema silviagrícola pode oscilar de favorecimento para competição, e viceversa, com estes estágios interativos coexistindo no tempo e espaço (Anderson \& Sinclair, 1993; Ong \& Huxley, 1996; Jose et al., 2008; Gea-Izquierdo et al., 2009). Contudo, tais resultados ainda são desconhecidos em sistemas silviagrícolas sob plantio direto nas condições subtropicais do Brasil, neste trabalho apresentamos a avaliação comparativa da produtividade do milho em um sistema silviagrícola e em monocultivo, com a qual se espera detectar os efeitos resultantes da interação (facilitação e competição)

\section{MATERIAL E MÉTODOS}

O experimento foi realizado na Estação Experimental Fazenda Modelo/IAPAR, em Ponta Grossa, Paraná $\left(25^{\circ} 07^{\prime} 22^{\prime \prime}\right.$ S; $50^{\circ} 03^{\prime} 01^{\prime \prime}$ W; e altitude de $953 \mathrm{~m})$. Duas áreas contíguas foram utilizadas: uma com 0,9 ha caracterizando o sistema agrícola (L) e outra, com 5,9 ha, caracterizando o sistema silviagrícola (iLF)). O solo das áreas é classificado como uma associação de Cambissolo Háplico Distrófico típico e Latossolo Vermelho Distrófico típico (Bognola \& Fasolo, 2003), em classe de relevo entre 4 e $9 \%$ de declividade com face de exposição Norte, e de baixa fertilidade natural. Amostras de solo da área experimental, coletadas em $11 / 08 / 2008$, apresentaram as seguintes características químicas: $\mathrm{pH}$ em $\mathrm{CaCl}_{2}$ : 5,43; $\mathrm{P}: 1,7 \mathrm{mg} \mathrm{dm}^{-3} ; \mathrm{K}$ : $0,10 \mathrm{cmol}_{\mathrm{C}} \mathrm{dm}^{-3}$; C: $11,62 \mathrm{~g} \mathrm{dm}^{-3}$; Al: $0,00 \mathrm{cmol}_{\mathrm{C}} \mathrm{dm}^{-3}$; Ca: $1,52 \mathrm{cmol}_{\mathrm{c}} \mathrm{dm}^{-3}$; Mg: $1,34 \mathrm{cmol}_{\mathrm{c}} \mathrm{dm}^{-3}$; T: 5,60 $\mathrm{cmol}_{\mathrm{C}} \mathrm{dm}^{-3}$; e V: $52,9 \% \%$. Os teores de argila, areia e silte são de 16,2; 79,3; e, 4,5, respectivamente.

O clima regional é classificado como $\mathrm{Cfb}$ subtropical mesotérmico úmido, de acordo com a classificação de Köeppen. A precipitação média entre 1.400 e $1.600 \mathrm{~mm}$ anuais, distribuída ao longo do ano, com leve declínio nos meses de abril a agosto (IAPAR, 1994).

Até o inverno de 2006, as áreas eram utilizadas para pastagem em uso convencional (extensivo) e estavam com vegetação de pouco valor forrageiro, quando foram preparadas, mediante aração, gradagem e incorporação de 3 ton./ha de calcário dolomítico, para o estabelecimento de um sistema silviagrícola que pudesse evoluir para um sistema agrossilvipastoril com árvores das espécies de eucalipto (Eucalyptus dunnii Maiden), aroeiravermelha (Schinus terebinthifolius Raddi) e grevílea (Grevillea robusta A. Cunn. ex R. Br.) plantadas em filas simples, com espaçamento de $14 \mathrm{~m} \times 3 \mathrm{~m}$, alocadas transversalmente ao sentido predominante da declividade do terreno para promoção do controle do escorrimento superficial das águas de chuva, e para que o deslocamento de máquinas e de animais fosse predominantemente transversal ao sentido da declividade. As espécies arbóreas foram dispostas alternadamente na mesma linha de plantio.

Desde então, a área vinha sendo utilizada para a produção de lavouras de inverno (aveia-preta - Avena strigosa Schreb) e lavouras de verão (soja Glycine max (L.) Merr.)

Em novembro de 2008 as dimensões médias das árvores eram de $9,2 \mathrm{~cm}$ de diâmetro à altura de $1,30 \mathrm{~m}$ do solo (DAP) e 4,8 $\mathrm{m}$ de altura total $(\mathrm{H})$ para o eucalipto, e alturas $(\mathrm{H})$ de $1,9 \mathrm{~m}$ e $1,2 \mathrm{~m}$ para a grevílea e aroeira-vermelha, respectivamente.

O plantio do milho foi realizado em $11 / 11 / 2008$, mediante plantio direto sobre palhada de aveia-preta dessecada com herbicida Glyfosate $(1,2 \mathrm{~kg}$ i.a./ha). O espaçamento entre linhas de miIho foi de $0,7 \mathrm{~m}$, com 5 sementes por metro linear, configurando uma população inicial, aproximadamente, de 71.500 plantas por hectare. A adubação de base foi de $220 \mathrm{~kg} \mathrm{ha}^{-1}$ de NPK na formulação 03:19:20 e a adubação em cobertura foi realizada aos 35 dias após emergência, na proporção de 67,5 $\mathrm{kg} \mathrm{ha}^{-1}$ de $\mathrm{N}$, em uma única aplicação de uréia para ambas as áreas ( $L$ e iLF).

$O$ delineamento experimental utilizado foi em blocos ao acaso com seis repetições e três tratamentos. Os tratamentos na condição silviagrícola corresponderam a duas posições entre dois renques arbóreos (T1 =adjacente aos renques e, T2 = central entre dois renques). $\mathrm{O}$ tratamento T3 correspondeu ao cultivo solteiro do milho (Testemunha).

A massa de grãos do milho foi avaliada em parcelas de $31,5 \mathrm{~m}^{2}(3,5 \mathrm{~m} \times 9,0 \mathrm{~m})$. As espigas foram colhidas manualmente e a trilhagem foi realizada por uma trilhadeira estacionária. Imediatamente após a trilha, foi feita a determinação do percentual de umidade para a amostra de cada parcela/ tratamento utilizando um determinador digital de umidade de grãos. Os dados de produtividade por parcela foram obtidos pela transformação da massa de grãos, corrigida para $14,5 \%$ de umidade (Sioga et al., 2009), obtida de cada amostra pela área da parcela. 
O incremento de madeira, durante o período da lavoura de milho, foi estimado a partir dos dados de DAP e $\mathrm{H}$ obtidos do monitoramento dendrométrico realizado mensalmente por meio da utilização de cintas dendrométricas (Botosso \& Tomazello Filho, 2001). O volume do tronco foi calculado usando a equação para o volume de cone:

$\square / 4$ * $\mathrm{DAP}^{2}{ }^{*} \mathrm{H}^{* 1 / 3} \quad[1]$

Os resultados das avaliações foram submetidos à análise de variância, após checagem da normalidade (teste Shapiro-Wilk), para testar o efeito das diferenças entre os tratamentos, considerando seis blocos $(G L=5)$. Havendo efeito significativo, a comparação, entre as médias dos tratamentos no silviagrícola e a média da testemunha, seria feita pelo teste de Dunnett a $5 \%$ de probabilidade e, para a comparação entre as medias dos tratamentos (T1 e T2) na condição silviagrícola seria feita pelo teste de Tukey a $5 \%$ de probabilidade. O software estatístico utilizado foi o Statistica ${ }^{\circledR}$ (Statsoft, 1996).

Para avaliar o efeito resultante da interação entre os componentes lavoura e árvores, foi utilizado a diferença entre a produtividade da lavoura em iLF e os seu rendimento em monocultivo (L), ou seja, a razão do efeito da interação (Kho, 2008): $\left.\square=\left(\mathrm{Y} 1_{\mathrm{iLF}}-\mathrm{Y} 1_{\mathrm{m}}\right) / \mathrm{Y} 1_{\mathrm{m}}\right) \quad$ [2], onde $\mathrm{Y}$ é o rendimento em unidades de produto por área, $1_{\mathrm{iLF}}$ é a espécie 1 de lavoura cultivada em iLF, e $1 \mathrm{~m}$ é a espécie 1 de lavoura cultivada em monocultivo.

$\square>0$ indica vantagem na produtividade do sistema iLF em relação ao rendimento da lavoura em monocultivo. Produtos da árvore, como madeira, frutos, sementes, etc. são considerados como adicionais ao rendimento da área.

\section{RESULTADOS E DISCUSSÃO}

No sistema silviagrícola o milho ocupou efetivamente $9,9 \pm 0,4 \mathrm{~m}$ de faixa plantada entre os renques, ou seja, média de $70,7 \%$ da área. As faixas de árvores (renques) ocuparam 4,1 $\pm 0,4 \mathrm{~m}$ de largura (29,3\% da área). Deste modo, as parcelas do tratamento T2 estavam $100 \%$ coberta com plantas de milho, enquanto que, as parcelas do tratamento T1 apresentavam $82,5 \%$ de suas áreas ocupadas com plantas de milho. A produtividade foi calculada levando em consideração esta diferença.

A análise de variância não detectou efeito significativo $(P>0,05)$ dos diferentes tratamentos na produtividade do milho (Tabela 1). No silviagrícola, a presença do componente arbóreo cobrindo parcialmente a área e interagindo com o componente lavoura, produz um efeito que é regido pelo equilíbrio dinâmico entre competição e facilitação (GeaIzquierdo et al., 2009). Por exemplo, a sombra das árvores pode favorecer as plantas de lavoura na medida em que diminui a temperatura de folha e o estresse térmico com efeitos sobre a fotossíntese; por outro lado, o sombreamento pode restringir a incidência de radiação fotossinteticamente ativa e também produzir efeitos sobre a fotossíntese. A intensidade de sombra que irá facilitar ou não a planta de lavoura, depende do arranjo e manejo dado às copas das árvores e das condições climáticas predominante na área durante o desenvolvimento da lavoura (Devkota et al., 2009; Brandle et al., 2004; Jose et al., 2000; Gilllespie et al, 2000)

Tabela 1. Produtividade do milho (var. IPR144) nos sistemas agrícola (L) e silviagrícola (iLF), nas condições edafoclimáticas dos Campos Gerais, Estação Experimental Fazenda Modelo/IAPAR, Ponta Grossa-PR. Safra 2008-09.

\begin{tabular}{|c|c|c|c|c|c|c|}
\hline \multicolumn{2}{|l|}{ Forma de uso da terra } & \multirow{2}{*}{$\frac{\mathrm{Kg} \mathrm{ha}^{-1}}{4.247,87}$} & \multirow{2}{*}{$\begin{array}{r}\text { CV\% } \\
14,77\end{array}$} & \multirow{2}{*}{ F-calc. } & \multirow{3}{*}{$\begin{array}{c}\mathrm{F}_{- \text {tab. }} \\
4,1028\end{array}$} & \multirow{3}{*}{$\begin{array}{r}p \text {-valor } \\
0,29447\end{array}$} \\
\hline Sistema agrícola $(\mathrm{L})$ & (T3) & & & & & \\
\hline \multirow{2}{*}{$\begin{array}{c}\text { Sistema silviagrícola } \\
\text { (iLF) }\end{array}$} & $\begin{array}{c}\text { meio da alameda } \\
\text { (T2) }\end{array}$ & $5.078,75$ & 30,82 & \multirow[t]{2}{*}{1,3849} & & \\
\hline & $\begin{array}{c}\text { adjacente ao } \\
\text { renque (T1) }\end{array}$ & $4.031,23$ & 25,58 & & & \\
\hline
\end{tabular}

Os valores da produtividade do milho amostrados em T1 e T2 (sistema silviagrícola) variaram grandemente (Tabela 1 ) em relação aos obtidos no T3 (Testemunha), indicando a natureza da heterogeneidade horizontal (Brutsaert, 1982) e de extensa área de interface (Young, 1994) existente no silviagrícola; cada hectare tem $0,706 \mathrm{~km}$ de linha de árvore, considerando os dois lados das linhas voltados para a faixa de lavoura de milho, a interface árvore-lavoura é de $1,412 \mathrm{~km} \mathrm{ha}^{-1}$. A heterogeneidade horizontal é dada pela presença de copas das árvores na linha e pela ausência na entre -linha (faixa de lavoura), podendo ser representada pela condição variante entre a mínima e a máxima cobertura de copas (o que é determinado pelo espaçamento e tamanho das árvores), implicando em forte dissimilaridade, tanto horizontal quanto vertical na distribuição de calor e, ou, vapor d'água (Spolador et al., 2006). Tais condições além de alterar a incidência de radiação solar para o plano do dossel da lavoura diminuem a velocidade dos ventos incidentes sobre a área (Tamang et al., 2010; 
PORFÍRIO-DA-SILVA, V. et al. Produtividade de milho em um sistema...

Brandle et al., 2004; Grala \& Colletti, 2003), com conseqüências sobre o déficit de pressão de vapor e balanço de energia (Tamang et al., 2010; Pezzopane et al., 2007), o que pode redundar em efeitos sobre a produtividade da lavoura

Nas áreas adjacentes da linha de árvores pode ocorrer aumento da competição por luz, água e nutrientes, somando a isto, pressões por alelopatia e plantas daninhas também podem ocorrer, afetando negativamente o rendimento de lavouras (Kuemmel, 2003; Ong \& Huxley, 1996). Contudo, nutrientes podem ser transferidos por meio da liteira ou de exsudatos radiculares (Dias et al., 2007; Young, 1994) para as lavouras favorecendo o seu desenvolvimento ou mitigando efeitos de competição. Esses efeitos, bem como a sua influência no rendimento ou na qualidade da produção, são espacialmente heterogêneos (Kuemmel, 2003).
Desde o plantio do milho $(11 / 11 / 08)$ até a colheita (18/04/09) foram 158 dias. A precipitação pluviométrica acumulou 196,0 mm em quatro eventos de chuvas, e o déficit hídrico atingiu $249,8 \mathrm{~mm}$ no período (Figura 1), conforme balanço hídrico normal decendial realizado pelo método de Thornthwaite \& Mather (Rolim et al., 1998). Segundo os dados de acompanhamento da safra 2008/09, a falta de chuvas ocorridas nos meses de novembro e dezembro/08 (Figura 1) foi prejudicial às lavouras de milho, pois estas se encontravam nas fases de floração e frutificação, o que ocasionou perdas (SEAB, 2009); a produtividade de milho na região dos Campos Gerais reduziu 18\% em relação à safra 2007/08 (SEAB, 2010).

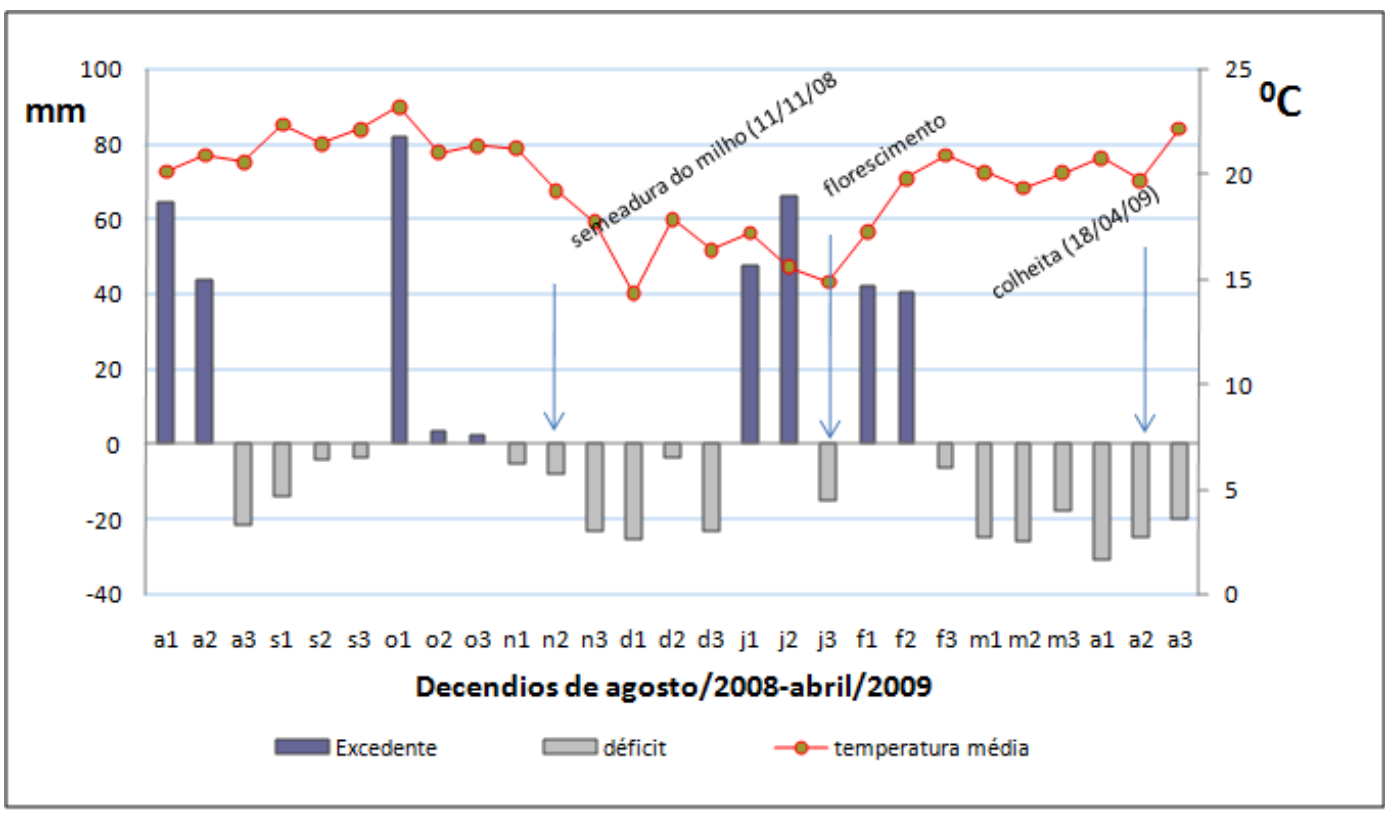

Figura 1. Extrato do balanço hídrico e temperatura média no período de outubro/2008 a abril/2009. Dados disponibilizados pelo Simepar- Estação 25135001- para a região de Ponta Grossa, PR.

Estudos demonstram que o efeito de proteção de quebra-ventos proporcionado pelas árvores e, ou, arbustos, favorecem a produtividade de lavouras (Brandle et al., 2004; Wright \& Brooks, 2002). Contudo, tal efeito positivo é mais dependente das espécies de lavoura e das condições climáticas reinantes durante o desenvolvimento destas no campo (Gea-lzquierdo et al., 2009; Nuberg et al., 2002). De modo geral, cereais sofrem com o sombreamento, mas certas vantagens podem ser observadas como o aumento de umidade proporcionado pela proteção de ventos em condições climáticas de seca (Kuemmel, 2003).

O rendimento médio do milho solteiro $(L)$ e no silviagrícola (iLF), foi de 4.247,9 $\pm 256,1 \mathrm{~kg} \mathrm{ha}^{-1} \mathrm{e}$ $4.554,99 \pm 409,9 \mathrm{~kg} \mathrm{ha}^{-1}$, respectivamente (Tabela
1). A razão do efeito da interação $(\square)$ entre os componentes lavoura e árvore foi de $6,7 \%$. Portando, sendo $\square>0$ (equação 2), a lavoura de milho no sistema silviagrícola (iLF) apresentou melhor desempenho do que no sistema agrícola $(L)$, provavelmente porque em iLF o impacto do veranico que se abateu no momento da antese foi menor, o que pode ser atribuído a proteção das arvores.

Tais rendimentos foram $36 \%$ e $32 \%$ menores do que rendimento médio, de $6.680 \mathrm{~kg} \mathrm{ha}^{-1}$, obtido na região dos Campos Gerais naquela safra (SEAB, 2010). Deve ser ressaltado que, o material genético de milho (Variedade IPR 114) utilizado, é menos produtivo do que material de milho híbrido que é plantado na quase totalidade das áreas produtoras de milho, o que contribui para a alta média 
PORFÍRIO-DA-SILVA, V. et al. Produtividade de milho em um sistema...

regional. Segundo Garcia \& Duarte (2006), o milho de sementes híbridas participa de $97 \%$ do mercado de sementes e são plantadas em $75 \%$ das áreas cultivadas com milho, enquanto que sementes de variedades participam com $3 \%$ do mercado e são plantadas em $25 \%$ das áreas.

Entretanto, no mesmo período da lavoura de milho, as árvores de eucalipto (33\% das árvores plantadas) cresceram 2,32 cm em diâmetro e 205 $\mathrm{cm}$ em altura, o que correspondeu a $1,03 \mathrm{~m}^{3} \mathrm{ha}^{-1} \mathrm{de}$ madeira acumulada em suplementação aos $4,5 \mathrm{mil}$ $\mathrm{kg} \mathrm{ha}^{-1}$ de milho obtidos no silviagrícola. Ao final da lavoura de milho, o estoque de madeira de eucalipto na área silviagrícola era de $1,87 \mathrm{~m}^{3} \mathrm{ha}^{-1}$.

Os incrementos da madeira das árvores, ou ainda outro produto florestal, (e.g.: frutos da aroeira, condimento conhecido como pimenta-rosa) são rendimentos adicionais do sistema iLF.

Mais estudos serão necessários para meIhor entendimento das interações (facilitação e, ou, competição) entre componentes árvore e lavouras ao longo do tempo, devido ao aumento das dimensões das árvores (tanto acima quanto abaixo do solo), o que oportunizará conhecimentos para estratégias diferenciadas de manejo, bem como da evolução da participação dos diferentes componentes no rendimento do sistema.

\section{AGRADECIMENTOS}

Aos funcionários da Estação Experimental Fazenda Modelo/lapar pelo suporte de campo para a realização deste trabalho.

\section{CONCLUSÕES}

A produtividade de grãos da lavoura de milho não foi negativamente afetada pela integração com árvores aos 29 meses de idade no arranjo espacial estudado.

O sistema silviagrícola favoreceu a lavoura de milho sob as condições climáticas reinantes na safra 2008/2009.

A avaliação de produtividade permite detectar a manifestação dos efeitos interativos de competição e facilitação (ou favorecimento) entre arvores e lavoura.

A causalidade dos efeitos, quer sejam: a restrição da radiação solar; o uso da água do solo; o uso de nutrientes; exsudatos de raízes; alterações do déficit de pressão de vapor, da velocidade do vento, da umidade relativa do ar, da evopotranspiração, da temperatura do ar, entre outras, devem ser alvo de estudos.

\section{REFERÊNCIAS}

1. ANDERSON, L. S.; SINCLAIR, F. L. Ecological interactions in agroforestry systems. Agroforestry Abstracts, v.6, n. 2, p. $57-$ 911993.

2. BOGNOLA, I. A.; FASOLO, P. J. Mapeamento dos solos e aptidão agrícola das terras da Fazenda Modelo - Ponta Grossa, PR. Embrapa Florestas. Colombo, PR, p. CD-ROM. 2003. (Relatório Final - Contrato de Coop. Técnica n.21500,02/0023-1). Não Publicado.

3. BOTOSSO, P. C.; TOMAZELLO FILHO, M. Aplicação de faixas dendrométricas na dendrocronologia: avaliação da taxa do ritmo de crescimento do tronco de árvores tropicais e subtropicais. In: MAIA, N. B.; MARTOS, H. L.; BARRELLA, W. [Org.] Indicadores ambientais: conceitos e aplicações. São Paulo: EDUC/COMPED/INEP, 2001. 285p. p.145-171.

4. BRANDLE, J. R.; HODGES, L.; ZHOU, X. H. Windbreaks in North American agricultural systems. Agroforestry Systems, v. 61, n. 1, p.65-78. 2004.

5. BRUTSAERT, W. Evaporation into the atmosphere. Theory, History, and Applications. Dordrecht: Kluwer Academic Publishers, 1991. 293 p. 3a. reimpressão.

6. BURNER, D. M.; POTE, D. H.; BELESKY, D. P. Effect of loblolly pine root pruning on alley cropped herbage production and tree growth. Agronomy Journal, v. 101, n. 1, p.184-192, 2009.

7. CALLAWAY, R. M.; WALKER, L. R. Competition and facilitation: A synthetic approach to interactions in plant communities Ecology, v. 78, n. 7, p.1958-1965, 1997.

8. DEVKOTA, N. R.; KEMP, P. D.; HODGSON, J.; VALENTINE, I.; JAYA, I. K. D. Relationship between tree canopy height and the production of pasture species in a silvopastoral system based on alder trees. Agroforestry Systems, n. 76, p.363$364,2009$.

9. DIAS, P.F.; SOUTO, S.M.; RESENDE, A.S.; URQUIAGA, S.; ROCHA, G.P.; MOREIRA, J.F.M.; FRANCO, A.A. Transferência do $\mathrm{N}$ fixado por leguminosas arbóreas para o capim Survenola crescido em consórcio. Ciência Rural [online]. 2007, vol. 37, no. 2, pp. 352-356. ISSN 0103-8478.

10.GARCIA, J. C.; DUARTE, J. O. Perspectiva do uso de sementes transgênicas na produção de milho no Brasil. In: Congresso Brasileiro de Economia e Sociologia Rural, 44, 2006, Fortaleza. Anais. Fortaleza: SOBER/BNB, 2006. Disponível em: <http://www.sober.org.br/ palestra/5/179.pdf>. Acesso em: 19 mar. 2010.

11.GEA-IZQUIERDO, G.; MONTERO, G; CANELLAS, I. Changes in limiting resources determine spatio-temporal variability in tree-grass interactions. Agroforestry Systems, n. 76, p. 375-387. 2009.

12.GILLESPIE, A. R.; JOSE, S.; MENGEL, D. B.; HOOVER, W. L.; POPE, P. E.; SEIFERT, J.R., BIEHLE, D.J.,STALL, T. BENJAMIN, T. J. Defining competition vectors in a temperate alley cropping system in the mid-western USA: 1 . Production physiology. Agroforestry Systems, n. 48, p. 25-40. 2000.

13.GRALA, R. K.; COLLETTI, J. P.. Estimates of additional Maize (Zea mays) yields required to offset costs of treewindbreaks in Midwestern USA. Agroforestry Systems, v. 59, n. 1, p.11-20. 2003.

14.IAPAR - Instituto Agronômico do Estado do Paraná. Cartas climáticas do Estado do Paraná 1994. Londrina, IAPAR, 1994. 49 p. ilust. (IAPAR, Documento, 18)

15.JOSE, S.; ALLEN, S. C.; NAIR, P. K. R. Tree-crop interactions: lessons from temperate alley-cropping systems. In: BATISH, D. R.; KOHLI, R. K.; JOSE, S.; SING, H. P. Ecological Basis of Agroforestry. Nova York: Crc Press - T \& F Group, 2008. p. 15-31. 382 p. 
PORFÍRIO-DA-SILVA, V. et al. Produtividade de milho em um sistema...

16.JOSE, S.; GILLESPIE, A. R.; SEIFERT, J. R.; BIEHLE, D. J. Defining competition vectors in a temperate alley cropping system in the Midwestern USA. 2- Competition for water. Agroforestry Systems. v.48, p.41-59, 2000.

17.KHO, R. M. Approaches to Tree-Environment-Crop Interactions. In: BATISH, D. R. et al. (Ed.) Ecological Basis of Agroforestry. Nova York: CRC Press - T \& F Groups, 2008. P.51-72.

18.KUEMMEL, B. Theoretical investigation of the effects of field margins and hedges on crops yields. Agriculture, Ecossystems \& Environment, n. 95, p. 287-392.

19.MILLER, A. W.; PALLARDY, S. G. Resource competition across the crop-tree interface in a maize-silver maple temperate alley cropping stand in Missouri. Agroforestry Systems, v. 53, n.3, p. 247-259. 2001.

20.NUBERG I. K.; MYLIUS S. J.; EDWARDS J. M.; DAVEY, C. Windbreak research in a South Australian cropping system. Australian Journal of Experimental Agriculture. v. 42, 2002. p. 781-795.

21.ONG, C.K; H. HUXLEY (Ed.) Tree - Crop Interactions. A Physiological approach. CAB INTERNATIONAL, Wallingford, UK, 1996. $385 \mathrm{p}$.

22.PEZZOPANE, J. R. M.; PEDRO JÚNIOR, M. P.; GALLO, P. B. Balanço de energia em cultivo de café a pleno sol e consorciado com banana 'Prata Anã'. Revista Brasileira de Agrometeorologia, Piracicaba, v.15, n.2, p.169-177, 2007

23.REIS, H. E.; MAGALHÃES, L. L.; OFUGI, C.; MELIDO, R. C. N. Agrossilvicultura no Cerrado, região noroeste do Estado de Minas Gerais. In: FERNANDES, E. N.; PACUILLO, D. S.; CASTRO, C. R. T.; MULLER, M. D.; ARCURI, P. B.; CARNEIRO, J. C. (Ed.) Sistemas Agrossilvipastoris na América do Sul: Desafios e potencialidades: Juiz de Fora, MG: Embrapa Gado de Leite, 2007. p.137-154

24.REYNOLDS, P. E.; SIMPSON, J. A.; THEVATHASAN, N. V.; GORDON, A. M. Effects of tree competition on corn and soybean photosynthesis, growth, and yield in a temperate tree-based agroforestry intercropping system in southern Ontario, Canada. Ecological Engineering, n.29, p.362-371. 2007.

25.ROLIM, G. S.; SENTELHAS, P. C.; BARBIERI, V. Planilhas no ambiente EXCEL ${ }^{\mathrm{TM}}$ para os cálculos de balanços hídricos: normal, seqüencial, de cultura e de produtividade real e potencial. Revista Brasileira de Agrometeorologia, Santa Maria, v. 6,n.1,p133-137, 1998.

26.SEAB. Secretaria de Estado da Agricultura e do Abastecimento do Paraná. Boletim Diário DERAL -2009 - milho. 2009. Disponível em: <http://www.seab.pr.gov.br/modules/qas/uploads/1892/milho_04fev2009.pdf>. Acesso em: 20 jan. 2010.

27.SEAB. Secretaria de Estado da Agricultura e do Abastecimento do Paraná. Estimativa de Safra. 2010. Disponível em: <http://www.seab.pr.gov.br/arquivos/File/deral/pss.xls>. Acesso em: 20 mar. 2010.

28.SIOGA, P. S.; GERAGE, A. C.; ARAUJO, P. M.; SERA, G. H. Avaliação estadual de cultivares de milho safra 2008/2009 Londrina: IAPAR, 2009. 65p. (Série Informe da Pesquisa, n. 157)

29.SPOLADOR, J.; SANCHES, L.; COSTA, M. H. Radiação fotossinteticamente ativa em uma floresta de transição CerradoAmazônica. Revista Brasileira de Meteorologia. v.21, n.3b, 301-307, 2006

30.STATSOFT, INC. Statistica for Windows 5.0. Computer program manual. Tulsa, OK: Statsoft, 1996.

31.TAMANG, B.; ANDREU, M. C.; ROCKWOOD, D. Microclimate patterns on the leeside of single-row tree windbreaks during weather conditions in Florida farms: implications for improved crop production. Agroforestry Systems, Online: 10/02/2010. 12p. Disponível em: <http://www.springerlink.com/content/j5j1h7826q073800>. Acesso em: 05 mar. 2010.

32.WRIGHT, A. J.; BROOKS, S. J. Effect of windbreaks on potato production for the Atherton Tablelands of North Queensland. Australian Journal of Experimental Agriculture. v. 42, p.797-807. 2002.

33.YOUNG, A. Agroforestry for soil conservation. 4. ed. Wellingdorf: CAB International, 1994. p.276

Recebido em 13/12/2011 Aceito em 25/02/2015 